\title{
Membrane Proteins Involved in Epithelial-Mesenchymal Transition and Tumor Invasion: Studies on TMPRSS4 and TM4SF5
}

\author{
Semi $\operatorname{Kim}^{1 *}$, Jung Weon Lee ${ }^{2 * *}$ \\ ${ }^{1}$ Immunotherapy Research Center, Korea Research Institute of Bioscience and Biotechnology, Daejeon 305-806, Korea, \\ ${ }^{2}$ Department of Pharmacy, College of Pharmacy, Seoul National University, Seoul 151-742, Korea
}

\begin{abstract}
The epithelial-mesenchymal transition (EMT) is one mechanism by which cells with mesenchymal features can be generated and is a fundamental event in morphogenesis. Recently, invasion and metastasis of cancer cells from the primary tumor are now thought to be initiated by the developmental process termed the EMT, whereby epithelial cells lose cell polarity and cell-cell interactions, and gain mesenchymal phenotypes with increased migratory and invasive properties. The EMT is believed to be an important step in metastasis and is implicated in cancer progression, although the influence of the EMT in clinical specimens has been debated. This review presents the recent results of two cell surface proteins, the functions and underlying mechanisms of which have recently begun to be demonstrated, as novel regulators of the molecular networks that induce the EMT and cancer progression.
\end{abstract}

Keywords: epithelial-mesenchymal transition, invasion, membrane proteins, TM4SF5, TMPRSS4

\section{Epithelial-Mesenchymal Transition}

Metastasis is the leading cause of cancer-related deaths in most cancer types. As an initial step in cancer metastasis, epithelial tumor cells in general disseminate from a primary solid tumor mass and invade into the surrounding stromal tissues. Invasion is enhanced by tumor cell activation of the epithelial-mesenchymal transition (EMT) [1-4]. The EMT is characterized by the loss of epithelial apicobasal polarity and cell-cell contacts, modulation of cell-matrix adhesion, enhanced proteolytic activity, cytoskeletal remodeling, and acquisition of the ability to migrate and invade the extracellular matrix (ECM) [1, 3]. During the EMT, epithelial cells undergo molecular changes; epithelial cells gradually lose their epithelial markers, such as E- cadherin, ZO-1, and cytokeratins, and concomitantly acquire mesenchymal markers, such as vimentin, fibronectin, $\mathrm{N}$ - cadherin, and alpha smooth muscle actin $[1,3]$. The EMT plays a critical role in the formation of various tissues and organs, such as the mesoderm, neural crest, heart, secondary palate, and peripheral nervous systems, during embryonic development and wound healing in adult organism $[2,4]$. Furthermore, the EMT is implicated in pathological processes, such as tumor cell invasion and metastasis and organ fibrosis [2].

One of the hallmarks of the EMT is the functional loss of E-cadherin, which is currently thought to be a metastasis suppressor [5]. Downregulation of E-cadherin is usually mediated by E-cadherin transcriptional repressors/EMTinducing transcription factors, including the Snail superfamily of zinc-finger factors (Snail and Slug), the ZEB family (ZEB1 and ZEB2), and basic helix-loop-helix factors (Twist1 and E47), which have been associated with tumor invasion and metastasis $[4,5]$. These factors repress transcription of E-cadherin by interacting with proximal E-box elements in the E-cadherin promoter [5]. In addition, these E-cadherin repressors may be directly or indirectly involved in the upregulation of certain mesenchymal genes [5], although the precise mechanism of this regulation is largely unknown.

The EMT is triggered by soluble growth factors, such as members of the transforming growth factor- $\beta$ (TGF $\beta)$ and 
fibroblast growth factor families, epidermal growth factor, and hepatocyte growth factor (HGF) $[3,4]$. Subsequent activation of receptor-mediated signaling triggers the activation of intracellular effector molecules, such as members of the small GTPase family, leading to changes in cytoskeletal organization, and also results in the activation of EMTinducing transcription factors [3, 4]. In addition, components of the ECM, such as collagen, and activation of integrin co-receptors are also involved in the EMT process [3]. Certain proteases are sufficient to induce the EMT [2]; for example, matrix metalloproteinase-3 triggers the EMT by increasing the cellular levels of reactive oxygen species, which in turn induces Snail expression [6].

Recently, microRNAs (miRs) have been identified as a novel class of EMT regulators; miRs that negatively regulate the EMT include miR-153, -155, -194, -25, -212, and -200, and miRs that positively regulate the EMT include miR-29a, $-103 / 107,-150$, and $-221 / 22$ [7]. miRs regulate invasion and metastasis by targeting the transcripts of various genes involved in the EMT event, including EMT-inducing transcription factors. For example, members of the miR-200 family are negative regulators of the EMT and are essential for maintenance of the epithelial status through the downregulation of ZEB1 and ZEB2. In turn, miR-200 members are transcriptionally repressed by ZEB1 and ZEB2, thus establishing a double-negative feedback loop [8].

The EMT was recently shown to be linked to stemness and self-renewal capacity $[9,10]$. In cases of breast cancer stems, the linkage among EMT phenotype, stemness, and drug resistance has been well studied [11]. Further, epithelialmesenchymal plasticity (consisting of EMT and MET) is also described in circulating tumor cells (CTCs) [12-14]. CTCs with various degrees of EMT phenotypes are found during breast cancer metastasis [15]. Therefore, CTCs may involve self-renewal capacity, which is linked to the EMT, during cancer metastasis [16].

\section{Transmembrane Protease Serine 4 (TMPRSS4)}

\section{Introduction to type II transmembrane serine proteases}

Dysregulation of proteases is a hallmark of cancer progression; thus, proteases in general have been the subject of numerous cancer studies. Extracellular proteolytic enzymes, including matrix metalloproteinases (MMPs) and serine proteases, contribute to tumor cell invasion and metastasis through both direct proteolytic activity and the regulation of cellular signaling and functions [17-19]. Most members of the serine protease family are either secreted or sequestered in cytoplasmic organelles awaiting signal-regulated release. Recently, type II transmembrane serine proteases (TTSPs) have been recognized as a new subfamily of serine proteases that have in common an extracellular proteolytic domain, a single-pass transmembrane domain, a short intracellular domain, and a variable-length stem region containing modular structural domains [20-24]. Enteropeptidase (also known as enterokinase), identified over a century ago due to its pivotal role in food digestion, is the first TTSP, which was revealed by molecular cloning of the enteropeptidase cDNA two decades ago [25]. TMPRSS2, human airway trypsin-like protease (HAT), corin, and matriptase have been subsequently identified as cell surface-associated proteases $[23,24]$. To date, 20 TTSPs have been identified in mouse and humans due to the analysis of sequence data from mammalian genome projects [23]. Analysis of the tissue distribution of TTSPs and gene targeting in mice of certain TTSPs suggested that a significant number of TTSPs may have important functions in embryonic development and homeostasis of mammalian tissues, such as heart, skin, inner ear, placenta, and digestive tract [23, 24].

Most TTSPs are overexpressed in a variety of tumors compared to normal tissues, implicating their potential as novel markers of tumor development and progression and possible molecular targets for anti-cancer therapeutics [23, 26]. Recently, a number of works have focused on the evaluation of the expression of individual TTSPs during tumor progression and on the investigation of the potential roles of these proteases in tumor cell proliferation, migration, and invasion [23, 27].

\section{TMPRSS4 in cancer}

TMPRSS4 (gene ID, 56649; chromosomal location, 11q23.3), initially referred to as TMPRSS3, was originally identified as a gene expressed in most pancreatic cancer tissues but not in the normal pancreas or chronic pancreatitis [28]. To date, 7 isoforms have been reported. The deduced sequence of 437 amino acids of the longest isoform (isoform 1) contains a serine protease domain with putative trypsin-like activity and a transmembrane domain [28]. In human, TMPRSS4 mRNA was detected in bladder, esophagus, stomach, small intestine, colon, and kidney [28], although the physiological roles of TMPRSS4 remain unknown. Furthermore, TMPRSS4 expression was upregulated in malignant compared to benign thyroid neoplasm and was suggested as both a diagnostic and prognostic marker [29, 30]. TMPRSS4 was associated with poor prognosis in non-small-cell lung cancer (NSCLC) with squamous cell histology [31], triple-negative breast cancer [32], cervical cancer [33], and gastric cancer patients [34]. Kim et al. [35] reported that TMPRSS4 mRNA levels were upregulated in colorectal cancer tissues versus adjacent normal mucosa. The authors also reported that TMPRSS4 protein 
expression was significantly higher in human colorectal cancer tissues from advanced stages $(52.5 \%$ and $50.0 \%$ of stages III and IV, respectively) than in that of early stage (6.3\% in stage I), suggesting that TMPRSS4 may play a role in the progression of non-invasive tumors to invasive malignancies [35]. Jia et al. [36] showed that the inhibitory tripeptide tyroserleutide led to downregulation of TMPRSS4 in hepatocellular carcinoma (HCC), thereby reducing the invasion and metastasis of HCC induced by irradiation. Taken together, TMPRSS4 may be a novel biomarker for the prognosis of certain types of cancers and could be employed for diagnostics and therapeutics.

On the other hand, the mechanism by which TMPRSS4 expression is modulated has not been well characterized. Recently, Nguyen et al. [37] reported that TMPRSS4 was increased in NSCLC cells under hypoxic conditions, suggesting that hypoxia within the tumor microenvironment may upregulate TMPRSS4 expression.

\section{Function of TMPRSS4 in the regulation of EMT and invasion}

In colon cancer cells, TMPRSS4 induces downregulation of E-cadherin and leads to EMT events, accompanying morphological changes and actin reorganization [38]. Suppression of TMPRSS4 by siRNA reduces cell invasion in colon and lung cancer cells, while overexpression TMPRSS4 induces migration, invasion, and metastasis [38]. Attachment and spreading of cells on the ECM, with concomitant formation of stress fibers and focal adhesions, is a prerequisite for cell migration. TMPRSS4 also modulates cell-matrix adhesion and cell spreading mainly through modulation of integrins, such as $\alpha 5 \beta 1$, which has been centrally implicated in the EMT and cell motility [39, 40], which probably contributes to enhanced motility and invasiveness. One of the molecular mechanisms by which TMPRSS4 mediates the EMT and invasiveness in tumor cells is that TMPRSS4 mediates focal adhesion kinase (FAK) signaling pathway activation and extracellular signalregulated kinase (ERK) activation, mainly through integrin $\alpha 5$ upregulation, leading to the EMT and invasiveness. Furthermore, TMPRSS4 overexpression in human colorectal cancer tissues positively correlates with enhanced expression of integrin $\alpha 5$ and inversely correlates with E-cadherin expression, confirming that TMPRSS4 modulates expression of EMT markers. Recently, Larzabal et al. [41] reported that miR-205 is involved in TMPRSS4-induced integrin $\alpha 5$ expression in NSCLC cells. To further implicate TMPRSS4 in the EMT, Cheng et al. [42] suggested that interactions between HGF activator inhibitor (HAI-1) and TMPRSS4 contribute to EMT events, including E-cadherin reduction and morphological changes in pancreatic cancer cells. In addition, TMPRSS4-induced E-cadherin reduction and EMT play a critical role in radiation-induced long-term metastasis of residual HCC in nude mice [43].

Interaction of TMPRSS 4 and integrin $\alpha 5$, based on the observation that TMPRSS4 partially interacts with integrin $\alpha 5$ under certain coimmunoprecipitation conditions in a cell line-dependent manner [35] (Kim S, unpublished observation), suggests the possibility that TMPRSS4 may modulate or participate in the interaction of integrin and other cell surface proteins (for example, tetraspanin, receptor tyrosine kinases, etc.), leading to subsequent signaling transduction activation. In fact, TMPRSS4 can interact with urokinase plasminogen activator receptor (uPAR; CD87) [44], which can induce the EMT in hypoxic breast cancer cells [45], although it is not clear whether TMPRSS4 interacts with uPAR directly or via integrin(s).

Loss or reduction of E-cadherin expression is a wellknown hallmark of the EMT and correlates positively with tumor cell invasion and metastasis [3]. TMPRSS4 appears to modulate SIP1/ZEB2 expression, based on the observation that SIP1 mRNA is upregulated in TMPRSS4-overexpressing colon cancer cells, although induction of SIP1 at the protein level remains to be determined. Therefore, it is possible that SIP1 mediates TMPRSS4-induced EMT events, including E-cadherin reduction.

Several studies have shown that suppression of high endogenous E-cadherin expression renders non-invasive cells partially invasive [46], whereas reconstitution of E-cadherin results in tumor cell reversion from an invasive mesenchymal phenotype to a benign epithelial phenotype $[46,47]$. In contrast, other studies have shown that ectopic expression of E-cadherin could not reverse EMT phenotypes induced by the transcription factor Twist1 [10]. On the other hand, downregulation of E-cadherin was required for TMPRSS4-mediated EMT and invasion in colon cancer cells but was not sufficient for induction of these phenotypes

TMPRSS4

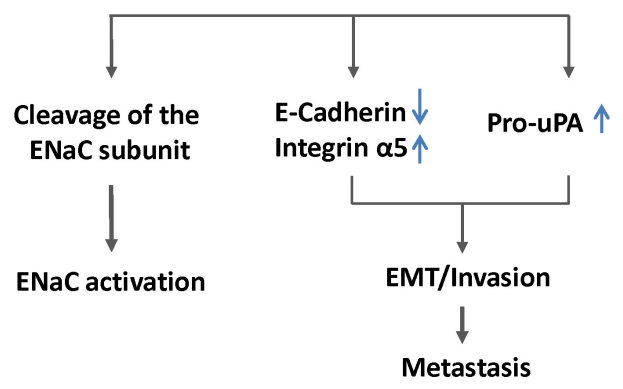

Fig. 1. Cellular functions of TMPRSS4. ENaC, epithelial sodium channel; uPA, urokinase plasminogen activator; EMT, epithelialmesenchymal transition. 
[35], suggesting that downregulation of E-cadherin is not the sole contributor to TMPRSS4-mediated phenotypes. In this respect, upregulation of specific mesenchymal markers, such as integrin $\alpha 5$, besides the downregulation of E-cadherin by TMPRSS4, may be required for full invasiveness during colon cancer progression (Fig. 1).

\section{Molecular mechanisms and signals regulated by TMPRSS4}

Numerous studies have focused on the investigation of in vivo substrates of TTSPs. However, few studies have conclusively addressed the in vivo molecular targets and function of TTSPs during tumor progression. In vitro, several TTSPs, including matriptase, were shown to activate prourokinase plasminogen activator, pro-macrophage stimulating protein-1, and pro-HGF, which are implicated in the proliferation, migration and invasion of various cancer cell types [23].

Like most of the members of the TTSP family, TMPRSS4 can activate epithelial sodium channel $(\mathrm{ENaC})$ in vitro through its proteolytic activity, possibly regulating sodium and water flux across high-resistance epithelia [48, 49]. TMPRSS4 induces cancer cell invasion in a manner that is dependent on serine proteolytic activity [38], and inhibitory compounds against TMPRSS4 serine protease activity were reported to reduce colon cancer cell invasion [50]. However, it remains unknown which precursor substrates are cleaved by TMPRSS4 to contribute to tumor progression. On the other hand, it has recently been reported that TMPRSS4 induces urokinase plasminogen activator (uPA) gene expression through activation of the transcription factors AP-1, Sp1, and Sp3 in mainly a JNK-dependent manner in prostate and lung cancer cells but not in colon cancer cells [44]. uPA is a well-known serine protease involved in invasion and metastasis and correlates with poor prognosis in breast, lung, stomach, bladder, colon, prostate, and ovarian cancers [51], and TMPRSS4 expression significantly correlates with UPA expression in human lung and prostate adenocarcinomas [44]. In addition, TMPRSS4-mediated uPA expression contributes to prostate cancer cell invasion (Fig. 1) [44]. It is intriguing that TMPRSS4 activates JNK signaling pathways, possibly through its association with uPAR, leading to uPA expression. uPAR can induce the EMT and stem cell-like properties in breast cancer cells by activating diverse cell signaling pathways, including ERK, phosphoinositide-3-kinase-Akt, and Racl [45, 52]. Therefore, the association of TMPRSS4 and UPAR and subsequent cell signaling modulation may be a novel mechanism for the control of invasion and the EMT.

The observations that TMPRSS4 modulates cell signaling and subsequently activates both AP-1 and Sp1/3 trans- criptional activities [44], which have been reported to be involved in the transcriptional regulation of the EMT and invasion [53], suggest that TMPRSS4 could modulate the expression of various genes, which may be associated with invasion and metastasis.

\section{Transmembrane 4 L Six Family Member 5 (TM4SF5)}

\section{The tetraspanins}

Tetraspanins (TM4SFs) have four transmembrane protein domains with two extracellular loops and one intracellular loop (ICL) and the $\mathrm{N}$ - and C-terminal tails [54]. They are expressed on the cell surface and/or intracellular vesicles and contain 33 members in mammals [55]. Tetraspanins, or TM4SFs, are suggested to be located at tetraspanin-enriched microdomains (TERMs) [56], where they form proteinprotein complexes in a homophilic or heterophilic manner with other TM4SFs, integrins, or growth factor receptors $[57,58]$. The protein complexes are known to regulate the dynamics of the complex components on the cell surface with regard to diffusion, trafficking, retention, and stability, in addition to influencing intracellular signal transductions $[56,59,60]$.

\section{TM4SF5 in cancer}

TM4SF5 (gene ID, 9032) maps to chromosome 17 at 17p13.3 according to Entrez Gene. In AceView, it covers $11.34 \mathrm{~kb}$, from 4621928 to 4633262 (NCBI 36, March 2006), on the direct strand containing 4 different gt-ag introns. Its transcription produces 2 alternatively spliced mRNAs via alternative polyadenylation sites, which putatively encode 2 different isoforms (197 and 132 amino acids), containing an L6 membrane domain (http://www.ncbi.nlm.nih.gov/IEB/ Research/Acembly/av.cgi?db=35g\&c=Gene\&l=TM4SF5). TM4SF5 $(20,823 \mathrm{Da})$ is a transmembrane glycoprotein; as a family group, it is related to the tetraspanin family (transmembrane $4 \mathrm{~L}$ six family), including TM4SF1 (L6, L6-Ag), TM4SF4 (IL-TIMP), TM4SF518 (L6D), and TM4SF20 [61, 62]. TM4SF5 is highly expressed in diverse types of cancers, including liver, pancreatic, gastric, colon, adrenocorticotropic hormone (corticotropin)-negative bronchial carcinoid tumors, soft-tissue sarcoma, nonendocrine lung, and papilla vateri carcinoma [63-66]. Similar to tetraspanins (i.e., transmembrane 4 superfamily, TM4SFs), TM4SF5 has four transmembrane domains (TM1-TM4), short cytoplasmic domains at their $\mathrm{N}$ - and C-termini, an ICL between TM2 and TM4, two extracellular loops, a smaller extracellular loop between TM1 and TM2, and a larger extracellular loop between TM3 and TM4 [61, 62]. Recent clinical studies separately report that TM4SF5 is highly 
expressed in tumors from deceased breast cancer patients, compared to those from 10-year breast cancer survivors [67], and that postoperative 5-year overall survival of esophageal cancer patients negatively correlates with TM4SF5 expression [68]. These reports suggest that TM4SF5 overexpression correlates with poor prognosis of cancer patients.

\section{TM4SF5-mediated regulation of signaling molecules}

TM4SF5 can appear to form TERMs on the cell surface, via formation of large protein-protein complexes with tetraspanins, integrins, and growth factor receptors [61, 69]. Therefore, by virtue of the protein complex formation, overexpressed TM4SF5 in cancer cells can influence or activate diverse intracellular signaling pathways for cell adhesion, proliferation, the EMT, migration, and invasion for tumor progression and maintenance.

TM4SF5 is shown to associate with integrins $\alpha 2, \beta 1[70$, 71], $\alpha 5$ [72], and epidermal growth factor receptor (EGFR) $[73,74]$ during cell migration [70, 71], angiogenesis [72], drug resistance [74], and fibrosis [73]. With association and retention of integrin $\alpha 5$ on the cell surface, TM4SF5 can activate intracellular signaling for FAK/c-Src activation, leading to STAT3 activity for vascular endothelial growth factor (VEGF) induction [72]. In addition, TM4SF5 directly interacts with FAK or c-Src to regulate migration [75] and invasive ECM degradation [76]. In addition, TM4SF5 expression causes AKT activation, which in turn causes phosphorylation of $\mathrm{p} 27^{\text {Kip1 }}$ Ser10 for its cytosolic translocation, where it can regulate RhoA activity for morphological change and migratory function [74].

\section{TM4SF5-mediated EMT in tumor progression}

TM4SF5 expression in hepatocytes or NSCLC leads to EMT phenotypes, which in turn cause loss of contact inhibition [74], enhance migration and invasion for metastasis [77], and render gefitinib resistance [78]. TM4SF5 expression causes morphological changes through abnormal regulation of RhoA and Rac1 in hepatocytes, together with the loss of E-cadherin expression leading to EMT induction [74] via induction of Slug [79]. Inhibition of TM4SF5mediated signaling events of the cytosolic enrichment of p2 $7^{\text {Kip1 }}$ abolishes abnormal multilayer cell growth [74] and retards the G1 to $S$ phase progression [80]. Further, inhibition of TM4SF5-mediated EMT by suppression of cytosolic p $27^{\text {Kip1 }}$ expression leads gefitinib-resistant NSCLC cells to become gefitinib-sensitive [78]. TM4SF5 is involved in activation of hepatic stellate cells by causing EMT processes, leading to a correlation with the development of liver fibrosis in CCl4-treated mouse models [81]. TM4SF5 expression is achieved by TGF 1 1-mediated Smad actions on EGFR activation [73], such that the important roles of the multifunctional cytokine TGF $\beta 1$ in the activation of hepatic stellate cells and the EMT are confirmed in a development of murine liver fibrosis. Since liver fibrosis can eventually lead to hepatocarcinoma at a high rate of over 70\% [82], the roles of TM4SF5 in the development of both fibrosis and tumorigenesis in the liver can be reasonable.

Meanwhile, TM4SF5 expression enhances directional migration and invasion of hepatocytes. TM4SF5 in hepatocytes causes directional migration at an enhanced speed and the formation of more invadosome-like structures enriched with cortactin, actin, and actin-regulatory proteins, like Arp2 and WASP [77]. TM4SF5-mediated directional migration involves direct interaction and activation of FAK via the ICL domain of TM4SF5 and the F1 lobe of the FAK FERM domain [75]. Further, TM4SF5-mediated invasive ECM degradation requires direct interaction between the $\mathrm{COOH}$-terminus of TM4SF5 and c-Src, which is linked to Tyr845 phosphorylation of EGFR to form more invasive protrusions [76]. TM4SF5-mediated multilayer growth [74], FAK activity, migration, and invasion [75] are abolished by an anti-TM4SF5 reagent, TSAHC (a synthetic compound), which appears to affect its N-glycosylation and at the same time block induction of the TM4SF5-dependent EMT phenotype and multilayer growth [83]. Therefore, TM4SF5 also plays important roles in tumor initiation and progression, possibly being supported by an EMT process.

\section{Other TM4SF5-mediated EMT-related biological processes}

The EMT is well known to be related also to the development [84] and stemness of self-renewal [9]. We also observed that TM4SF5 can play roles in other EMT-mediated biological processes, like development of muscles and self-renewal of cancer cells. In zebrafish, suppression of tm $4 s f 5$ results in abnormal development, with an aberrant trunk and morphology of muscle fibers, presumably via alterations in the expression and localization of integrin $\alpha 5$, which is necessary for somite boundary maintenance (Choi $\mathrm{YJ}$ and Lee JW, in revision). In addition to liver fibrosis and tumorigenesis, therefore, TM4SF5 expression is importantly involved in the development of zebrafish muscles, which might bemediated by EMT.

Presumably, these diverse cellular effects by TM4SF 5 expression might be possible due to the characteristics of TM4SF5 - similar to tetraspanins - which forms large protein networks via heterophilic or homophilic interactions between tetraspanins, integrins, and growth factor receptors. TM4SF5 is shown to bind integrin $\alpha 2, \beta 1[70,71], \alpha 5$ [72], EGFR [73], and interleukin 6 receptor (IL6R) (Ryu J and Lee JW, in revision). Although its ligand has not been 
identified, interaction(s) with (an)other membrane protein or receptor can recapitulate the ligand binding-based activation. Therefore, TM4SF5 can transduce signaling activities for diverse cellular functions, including the EMT and different EMT-mediated phenotypes. Although diverse miRs are known to regulate the EMT [7], miRs targeting TM4SF5 are being studied.

\section{TM4SF5-mediated gene regulation}

Comparison of protein expression patterns between TM4SF5-null and -expressing cells shows a negative correlation between TM4SF5 and cell-cell adhesion-related molecules of epithelial markers, including E-cadherin [74], and a positive correlation between TM4SF5 and mesenchymal markers, including Slug [79], supporting TM4SF5- mediated EMT. Reverse transcription polymerase chain reaction analyses of show that TM4SF5-mediated regulation of their expressions occurs at the transcriptional level (Lee JW, unpublished observation). However, the signaling pathways underlying this regulation have not been determined yet.

In addition, TM4SF5 expression also correlates with cytosolic p2 $7^{\text {Kip1 }}$ [74]. Although p27 $7^{\text {Kip1 }}$ in the nucleus is inhibitory to cyclin-dependent kinases, suppressing the cell cycle and proliferation, its localization in the cytosol can lead to tumorigenic functions [85]. Cytosolic $\mathrm{p} 27^{\mathrm{Kip} 1}$ has been reported in different clinical reports, where different cancer types show enriched cytosolic localization of $\mathrm{p} 27^{\mathrm{Kip} 1}$ [86-88], suggesting that cytosolic $\mathrm{p} 27^{\mathrm{Kip} 1}$ can be tumorigenic [89]. p2 $7^{\text {Kip } 1}$ can be phosphorylated by Akt, KIS, or JNK [90-92], resulting in translocalization and stabilization in the cytosol, where it binds to and inactivates RhoA GTPase, leading to alterations in actin organization and motility regulation [93]. TM4SF5 expression also causes overexpression of p2 $7^{\text {Kip } 1}$, although how it occurs is unknown; TM4SF5 causes Akt-mediated Ser10 phosphorylation of $\mathrm{p} 27^{\mathrm{Kip} 1}$, leading to its stabilization, RhoA activity changes, and eventually morphological elongation for the EMT and contact inhibition loss [74]. JNK-mediated $\mathrm{p} 27^{\text {Kip1 }}$ phosphorylation in a TM4SF5-dependent manner also results in localization of p2 $7^{\text {Kip1 }}$ at cell-cell contacts [91], possibly leading to altered actin organization at cell-cell contacts. In addition, inhibition of the proteasome in terms of activity and subunit expression also depends on TM4SF5 expression, resulting in morphological changes and the EMT, suggesting another novel mechanism for TM4SF5-mediated EMT [79].

Meanwhile, TM4SF5 causes activation of the FAK/c-Src signaling pathways, leading to STAT3 phosphorylation at Tyr705 for the induction and secretion of VEGF, which can stimulate neighboring endothelial cells for enhanced (tumor) angiogenesis [72]. During modeling of the tumor

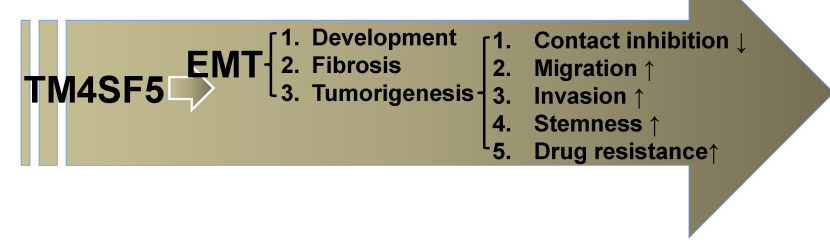

Fig. 2. TM4SF5-mediated epithelial-mesenchymal transition (EMT) is involved in diverse cellular functions, leading to liver tumorigenesis and maintenance in addition to developmental processes.

microenvironment, cancer cells overexpressing TM4SF5 appear to negatively regulate expression of the cytokine IL6, and exogenous IL6 treatment leads to less STAT3 signaling activation in TM4SF5-positive cancer cells (Ryu J and Lee JW, in revision); thus, TM4SF5-dependent suppression of IL6 can be a strategy for TM4SF5-positive tumor cells to avoid pro-immunological actions by IL6 secreted by neighboring immune cells. As for invasion, TM4SF5 expression also increases the mRNA and protein levels of MMP2, in addition to its activity [77].

Therefore, TM4SF5 expression correlates with or plays important roles in tumorigenesis in different mechanisms, including induction of the EMT and gene regulation (Fig. 2).

\section{Concluding Remarks}

Considering that such membrane proteins as TMPRSS4 or TM4SF5 may be important upstream regulators of the EMT and the invasiveness of cancer cells and because their expression differs substantially in normal and cancer tissues, targeting them could be a novel therapeutic strategy for the treatment of cancer metastasis. In the future, the functional involvement of TMPRSS4 and/or TM4SF5 in the initiation and progression of tumors needs to be evaluated using mouse models. Cancer-associated mutations and singlenucleotide polymorphisms within TMPRSS4 or TM4SF5 also need to be analyzed in association with cancer risk.

\section{References}

1. Kalluri R. EMT: when epithelial cells decide to become mesenchymal-like cells. J Clin Invest 2009;119:1417-1419.

2. Thiery JP, Acloque H, Huang RY, Nieto MA. Epithelial-mesenchymal transitions in development and disease. Cell 2009; 139:871-890.

3. Thiery JP, Sleeman JP. Complex networks orchestrate epithelial-mesenchymal transitions. Nat Rev Mol Cell Biol 2006; 7:131-142.

4. Yang J, Weinberg RA. Epithelial-mesenchymal transition: at the crossroads of development and tumor metastasis. Dev Cell 2008;14:818-829. 
5. Peinado H, Olmeda D, Cano A. Snail, Zeb and bHLH factors in tumour progression: an alliance against the epithelial phenotype? Nat Rev Cancer 2007;7:415-428.

6. Radisky DC, Levy DD, Littlepage LE, Liu H, Nelson CM, Fata $\mathrm{JE}$, et al. Raclb and reactive oxygen species mediate MMP-3-induced EMT and genomic instability. Nature 2005; 436:123-127.

7. Bouyssou JM, Manier S, Huynh D, Issa S, Roccaro AM, Ghobrial IM. Regulation of microRNAs in cancer metastasis. Biochim Biophys Acta 2014;1845:255-265.

8. Brabletz S, Brabletz T. The ZEB/miR-200 feedback loop: a motor of cellular plasticity in development and cancer? EMBO Rep 2010;11:670-677.

9. Mani SA, Guo W, Liao MJ, Eaton EN, Ayyanan A, Zhou AY, et al. The epithelial-mesenchymal transition generates cells with properties of stem cells. Cell 2008;133:704-715.

10. Yang J, Mani SA, Donaher JL, Ramaswamy S, Itzykson RA, Come $\mathrm{C}$, et al. Twist, a master regulator of morphogenesis, plays an essential role in tumor metastasis. Cell 2004;117:927939.

11. Pinto CA, Widodo E, Waltham M, Thompson EW. Breast cancer stem cells and epithelial mesenchymal plasticity: implications for chemoresistance. Cancer Lett 2013;341:56-62.

12. Armstrong AJ, Marengo MS, Oltean S, Kemeny G, Bitting RL, Turnbull JD, et al. Circulating tumor cells from patients with advanced prostate and breast cancer display both epithelial and mesenchymal markers. Mol Cancer Res 2011;9:997-1007.

13. Bednarz-Knoll N, Alix-Panabieres C, Pantel K. Plasticity of disseminating cancer cells in patients with epithelial malignancies. Cancer Metastasis Rev 2012;31:673-687.

14. Rhim AD, Mirek ET, Aiello NM, Maitra A, Bailey JM, McAllister F, et al. EMT and dissemination precede pancreatic tumor formation. Cell 2012;148:349-361.

15. Yu M, Bardia A, Wittner BS, Stott SL, Smas ME, Ting DT, et al. Circulating breast tumor cells exhibit dynamic changes in epithelial and mesenchymal composition. Science 2013;339: 580-584.

16. Tiwari N, Gheldof A, Tatari M, Christofori G. EMT as the ultimate survival mechanism of cancer cells. Semin Cancer Biol 2012;22:194-207.

17. Deryugina EI, Quigley JP. Matrix metalloproteinases and tumor metastasis. Cancer Metastasis Rev 2006;25:9-34.

18. Duffy MJ. Proteases as prognostic markers in cancer. Clin Cancer Res 1996;2:613-618.

19. Stetler-Stevenson WG, Yu AE. Proteases in invasion: matrix metalloproteinases. Semin Cancer Biol 2001;11:143-152.

20. Bugge TH, Antalis TM, Wu Q. Type II transmembrane serine proteases. J Biol Chem 2009;284:23177-23181.

21. Hooper JD, Clements JA, Quigley JP, Antalis TM. Type II transmembrane serine proteases. Insights into an emerging class of cell surface proteolytic enzymes. J Biol Chem 2001;276: 857-860.

22. Netzel-Arnett S, Hooper JD, Szabo R, Madison EL, Quigley JP, Bugge $\mathrm{TH}$, et al. Membrane anchored serine proteases: a rapidly expanding group of cell surface proteolytic enzymes with potential roles in cancer. Cancer Metastasis Rev 2003;22:237258.
23. Szabo R, Bugge TH. Type II transmembrane serine proteases in development and disease. Int J Biochem Cell Biol 2008;40: 1297-1316.

24. Szabo R, Wu Q, Dickson RB, Netzel-Arnett S, Antalis TM, Bugge TH. Type II transmembrane serine proteases. Thromb Haemost 2003;90:185-193.

25. Kitamoto Y, Yuan X, Wu Q, McCourt DW, Sadler JE. Enterokinase, the initiator of intestinal digestion, is a mosaic protease composed of a distinctive assortment of domains. Proc Natl Acad Sci U S A 1994;91:7588-7592.

26. Adler J, Parmryd I. Quantifying colocalization by correlation: the Pearson correlation coefficient is superior to the Mander's overlap coefficient. Cytometry A 2010;77:733-742.

27. Ohler A, Becker-Pauly C. TMPRSS4 is a type II transmembrane serine protease involved in cancer and viral infections. Biol Chem 2012;393:907-914.

28. Wallrapp C, Hähnel S, Müller-Pillasch F, Burghardt B, Iwamura $\mathrm{T}$, Ruthenbürger $\mathrm{M}$, et al. A novel transmembrane serine protease (TMPRSS3) overexpressed in pancreatic cancer. Cancer Res 2000;60:2602-2606.

29. Kebebew E, Greenspan FS, Clark OH, Woeber KA, Grunwell J. Extent of disease and practice patterns for medullary thyroid cancer. J Am Coll Surg 2005;200:890-896.

30. Kebebew E, Peng M, Reiff E, Treseler P, Woeber KA, Clark OH, et al. A phase II trial of rosiglitazone in patients with thyroglobulin-positive and radioiodine-negative differentiated thyroid cancer. Surgery 2006;140:960-966.

31. Larzabal L, Nguewa PA, Pio R, Blanco D, Sanchez B, Rodríguez MJ, et al. Overexpression of TMPRSS4 in non-small cell lung cancer is associated with poor prognosis in patients with squamous histology. Br J Cancer 2011;105:1608-1614.

32. Cheng D, Kong H, Li Y. TMPRSS4 as a poor prognostic factor for triple-negative breast cancer. Int J Mol Sci 2013;14:1465914668.

33. Cheng D, Kong H, Li Y. Prognostic value of interleukin-8 and MMP-9 in nasopharyngeal carcinoma. Eur Arch Otorhinolaryngol 2014;271:503-509.

34. Luo ZY, Wang YY, Zhao ZS, Li B, Chen JF. The expression of TMPRSS4 and Erk1 correlates with metastasis and poor prognosis in Chinese patients with gastric cancer. PLoS One 2013; 8:e70311.

35. Kim S, Kang HY, Nam EH, Choi MS, Zhao XF, Hong CS, et al. TMPRSS4 induces invasion and epithelial-mesenchymal transition through upregulation of integrin alpha5 and its signaling pathways. Carcinogenesis 2010;31:597-606.

36. Jia JB, Wang WQ, Sun HC, Liu L, Zhu XD, Kong LQ, et al. A novel tripeptide, tyroserleutide, inhibits irradiation-induced invasiveness and metastasis of hepatocellular carcinoma in nude mice. Invest New Drugs 2011;29:861-872.

37. Nguyen TH, Weber W, Havari E, Connors T, Bagley RG, McLaren R, et al. Expression of TMPRSS4 in non-small cell lung cancer and its modulation by hypoxia. Int J Oncol 2012; 41:829-838.

38. Jung H, Lee KP, Park SJ, Park JH, Jang YS, Choi SY, et al. TMPRSS4 promotes invasion, migration and metastasis of human tumor cells by facilitating an epithelial-mesenchymal transition. Oncogene 2008;27:2635-2647. 
39. Maschler S, Wirl G, Spring H, Bredow DV, Sordat I, Beug H, et al. Tumor cell invasiveness correlates with changes in integrin expression and localization. Oncogene 2005;24:2032-2041.

40. Nam EH, Lee Y, Park YK, Lee JW, Kim S. ZEB2 upregulates integrin alpha5 expression through cooperation with Sp1 to induce invasion during epithelial-mesenchymal transition of human cancer cells. Carcinogenesis 2012;33:563-571.

41. Larzabal L, de Aberasturi AL, Redrado M, Rueda P, Rodriguez MJ, Bodegas ME, et al. TMPRSS4 regulates levels of integrin alpha5 in NSCLC through miR-205 activity to promote metastasis. Br J Cancer 2014;110:764-774.

42. Cheng H, Fukushima T, Takahashi N, Tanaka H, Kataoka H. Hepatocyte growth factor activator inhibitor type 1 regulates epithelial to mesenchymal transition through membranebound serine proteinases. Cancer Res 2009;69:1828-1835.

43. Li T, Zeng ZC, Wang L, Qiu SJ, Zhou JW, Zhi XT, et al. Radiation enhances long-term metastasis potential of residual hepatocellular carcinoma in nude mice through TMPRSS4-induced epithelial-mesenchymal transition. Cancer Gene Ther 2011;18:617-626.

44. Min HJ, Lee Y, Zhao XF, Park YK, Lee MK, Lee JW, et al. TMPRSS4 upregulates uPA gene expression through JNK signaling activation to induce cancer cell invasion. Cell Signal 2014;26:398-408.

45. Lester RD, Jo M, Montel V, Takimoto S, Gonias SL. uPAR induces epithelial-mesenchymal transition in hypoxic breast cancer cells. J Cell Biol 2007;178:425-436.

46. Vleminckx K, Vakaet L Jr, Mareel M, Fiers W, van Roy F. Genetic manipulation of E-cadherin expression by epithelial tumor cells reveals an invasion suppressor role. Cell 1991;66: 107-119.

47. Cavallaro U, Christofori G. Cell adhesion and signalling by cadherins and Ig-CAMs in cancer. Nat Rev Cancer 2004;4: 118-132.

48. Andreasen D, Vuagniaux G, Fowler-Jaeger N, Hummler E, Rossier BC. Activation of epithelial sodium channels by mouse channel activating proteases (mCAP) expressed in Xenopus oocytes requires catalytic activity of mCAP3 and mCAP2 but not mCAP1. J Am Soc Nephrol 2006;17:968-976.

49. Vuagniaux G, Vallet V, Jaeger NF, Hummler E, Rossier BC. Synergistic activation of $\mathrm{ENaC}$ by three membrane-bound channel-activating serine proteases (mCAP1, mCAP2, and mCAP3) and serum- and glucocorticoid-regulated kinase (Sgk1) in Xenopus Oocytes. J Gen Physiol 2002;120:191-201.

50. Kang S, Min HJ, Kang MS, Jung MG, Kim S. Discovery of novel 2-hydroxydiarylamide derivatives as TMPRSS4 inhibitors. Bioorg Med Chem Lett 2013;23:1748-1751.

51. Andreasen PA, Kjøller L, Christensen L, Duffy MJ. The urokinase-type plasminogen activator system in cancer metastasis: a review. Int J Cancer 1997;72:1-22.

52. Jo M, Eastman BM, Webb DL, Stoletov K, Klemke R, Gonias SL. Cell signaling by urokinase-type plasminogen activator receptor induces stem cell-like properties in breast cancer cells. Cancer Res 2010;70:8948-8958.

53. Fuxe J, Vincent T, Garcia de Herreros A. Transcriptional crosstalk between TGF-beta and stem cell pathways in tumor cell invasion: role of EMT promoting Smad complexes. Cell Cycle
2010;9:2363-2374.

54. Sala-Valdés M, Ailane N, Greco C, Rubinstein E, Boucheix C. Targeting tetraspanins in cancer. Expert Opin Ther Targets 2012;16:985-997.

55. Detchokul S, Williams ED, Parker MW, Frauman AG. Tetraspanins as regulators of the tumour microenvironment: implications for metastasis and therapeutic strategies. $\mathrm{Br} J$ Pharmacol 2013 Jun 3 [Epub]. http://dx.doi.org/10.1111/ bph. 12260.

56. Yáñez-Mó M, Barreiro O, Gordon-Alonso M, Sala-Valdés M, Sánchez-Madrid F. Tetraspanin-enriched microdomains: a functional unit in cell plasma membranes. Trends Cell Biol 2009;19:434-446.

57. Berditchevski F. Complexes of tetraspanins with integrins: more than meets the eye. J Cell Sci 2001;114(Pt 23): 4143-4151.

58. Stipp CS, Kolesnikova TV, Hemler ME. Functional domains in tetraspanin proteins. Trends Biochem Sci 2003;28:106-112.

59. Berditchevski F, Odintsova E. Tetraspanins as regulators of protein trafficking. Traffic 2007;8:89-96.

60. Rubinstein E. The complexity of tetraspanins. Biochem Soc Trans 2011;39:501-505.

61. Lee SA, Park KH, Lee JW. Modulation of signaling between TM4SF5 and integrins in tumor microenvironment. Front Biosci (Landmark Ed) 2011;16:1752-1758.

62. Wright MD, Ni J, Rudy GB. The L6 membrane proteins: a new four-transmembrane superfamily. Protein Sci 2000;9:15941600 .

63. Gress TM, Wallrapp C, Frohme M, Müller-Pillasch F, Lacher $\mathrm{U}$, Friess $\mathrm{H}$, et al. Identification of genes with specific expression in pancreatic cancer by cDNA representational difference analysis. Genes Chromosomes Cancer 1997;19:97-103.

64. Kaneko R, Tsuji N, Kamagata C, Endoh T, Nakamura M, Kobayashi D, et al. Amount of expression of the tumor-associated antigen L6 gene and transmembrane 4 superfamily member 5 gene in gastric cancers and gastric mucosa. Am J Gastroenterol 2001;96:3457-3458.

65. Müller-Pillasch F, Wallrapp C, Lacher U, Friess H, Büchler M, Adler $\mathrm{G}$, et al. Identification of a new tumour-associated antigen TM4SF5 and its expression in human cancer. Gene 1998; 208:25-30.

66. Pascual-Le Tallec L, Dulmet E, Bertagna X, de Keyzer Y. Identification of genes associated with the corticotroph phenotype in bronchial carcinoid tumors. J Clin Endocrinol Metab 2002;87:5015-5022.

67. Karlsson E, Delle U, Danielsson A, Olsson B, Abel F, Karlsson $\mathrm{P}$, et al. Gene expression variation to predict 10-year survival in lymph-node-negative breast cancer. BMC Cancer 2008;8:254.

68. Wu YB, Huang YS, Xu YP, Sun YF, Yu DL, Zhang XQ, et al. A high level of TM4SF5 is associated with human esophageal cancer progression and poor patient survival. Dig Dis Sci 2013;58:2623-2633.

69. Hemler ME. Tetraspanin proteins mediate cellular penetration, invasion, and fusion events and define a novel type of membrane microdomain. Annu Rev Cell Dev Biol 2003;19: 397-422.

70. Lee SA, Kim YM, Kwak TK, Kim HJ, Kim S, Ko W, et al. The ex- 
tracellular loop 2 of TM4SF5 inhibits integrin alpha2 on hepatocytes under collagen type I environment. Carcinogenesis 2009;30:1872-1879.

71. Lee SY, Kim YT, Lee MS, Kim YB, Chung E, Kim S, et al. Focal adhesion and actin organization by a cross-talk of TM4SF5 with integrin alpha 2 are regulated by serum treatment. Exp Cell Res 2006;312:2983-2999.

72. Choi S, Lee SA, Kwak TK, Kim HJ, Lee MJ, Ye SK, et al. Cooperation between integrin alpha 5 and tetraspan TM4SF5 regulates VEGF-mediated angiogenic activity. Blood 2009;113: 1845-1855.

73. Kang M, Choi S, Jeong SJ, Lee SA, Kwak TK, Kim H, et al. Cross-talk between TGFbeta1 and EGFR signalling pathways induces TM4SF5 expression and epithelial-mesenchymal transition. Biochem J 2012;443:691-700.

74. Lee SA, Lee SY, Cho IH, Oh MA, Kang ES, Kim YB, et al. Tetraspanin TM4SF5 mediates loss of contact inhibition through epithelial-mesenchymal transition in human hepatocarcinoma. J Clin Invest 2008;118:1354-1366.

75. Jung O, Choi S, Jang SB, Lee SA, Lim ST, Choi YJ, et al. Tetraspan TM4SF5-dependent direct activation of FAK and metastatic potential of hepatocarcinoma cells. J Cell Sci 2012; 125(Pt 24):5960-5973.

76. Jung O, Choi YJ, Kwak TK, Kang M, Lee MS, Ryu J, et al. The $\mathrm{COOH}$-terminus of TM4SF5 in hepatoma cell lines regulates c-Src to form invasive protrusions via EGFR Tyr845 phosphorylation. Biochim Biophys Acta 2013;1833:629-642.

77. Lee SA, Kim TY, Kwak TK, Kim H, Kim S, Lee HJ, et al. Transmembrane $4 \mathrm{~L}$ six family member 5 (TM4SF5) enhances migration and invasion of hepatocytes for effective metastasis. J Cell Biochem 2010;111:59-66.

78. Lee MS, Kim HP, Kim TY, Lee JW. Gefitinib resistance of cancer cells correlated with TM4SF5-mediated epithelial-mesenchymal transition. Biochim Biophys Acta 2012;1823:514-523.

79. Kim JY, Nam JK, Lee SA, Lee MS, Cho SK, Park ZY, et al. Proteasome inhibition causes epithelial-mesenchymal transition upon TM4SF5 expression. J Cell Biochem 2011;112:782792.

80. Kim H, Kang M, Lee SA, Kwak TK, Jung O, Lee HJ, et al. TM4SF5 accelerates G1/S phase progression via cytosolic p27Kip1 expression and RhoA activity. Biochim Biophys Acta 2010;1803:975-982.

81. Kang M, Jeong SJ, Park SY, Lee HJ, Kim HJ, Park KH, et al. Antagonistic regulation of transmembrane $4 \mathrm{~L} 6$ family member 5 attenuates fibrotic phenotypes in $\mathrm{CCl}(4)$-treated mice.
FEBS J 2012;279:625-635.

82. Sangiovanni A, Del Ninno E, Fasani P, De Fazio C, Ronchi G, Romeo R, et al. Increased survival of cirrhotic patients with a hepatocellular carcinoma detected during surveillance. Gastroenterology 2004;126:1005-1014.

83. Lee SA, Ryu HW, Kim YM, Choi S, Lee MJ, Kwak TK, et al. Blockade of four-transmembrane L6 family member 5 (TM4SF5)-mediated tumorigenicity in hepatocytes by a synthetic chalcone derivative. Hepatology 2009;49:1316-1325.

84. Cannito S, Novo E, di Bonzo LV, Busletta C, Colombatto S, Parola M. Epithelial-mesenchymal transition: from molecular mechanisms, redox regulation to implications in human health and disease. Antioxid Redox Signal 2010;12:1383-1430.

85. Besson A, Dowdy SF, Roberts JM. CDK inhibitors: cell cycle regulators and beyond. Dev Cell 2008;14:159-169.

86. Baldassarre G, Belletti B, Bruni P, Boccia A, Trapasso F, Pentimalli F, et al. Overexpressed cyclin D3 contributes to retaining the growth inhibitor p27 in the cytoplasm of thyroid tumor cells. J Clin Invest 1999;104:865-874.

87. Cordon-Cardo C, Koff A, Drobnjak M, Capodieci P, Osman I, Millard SS, et al. Distinct altered patterns of p27KIP1 gene expression in benign prostatic hyperplasia and prostatic carcinoma. J Natl Cancer Inst 1998;90:1284-1291.

88. Hidaka T, Hama S, Shrestha P, Saito T, Kajiwara Y, Yamasaki F, et al. The combination of low cytoplasmic and high nuclear expression of $\mathrm{p} 27$ predicts a better prognosis in high-grade astrocytoma. Anticancer Res 2009;29:597-603.

89. Chu IM, Hengst L, Slingerland JM. The Cdk inhibitor p27 in human cancer: prognostic potential and relevance to anticancer therapy. Nat Rev Cancer 2008;8:253-267.

90. Fujita N, Sato S, Tsuruo T. Phosphorylation of p27Kip1 at threonine 198 by $\mathrm{p} 90$ ribosomal protein $\mathrm{S} 6$ kinases promotes its binding to 14-3-3 and cytoplasmic localization. J Biol Chem 2003;278:49254-49260.

91. Kim H, Jung O, Kang M, Lee MS, Jeong D, Ryu J, et al. JNK signaling activity regulates cell-cell adhesions via TM4SF5mediated p27(Kip1) phosphorylation. Cancer Lett 2012;314: 198-205.

92. Liang J, Zubovitz J, Petrocelli T, Kotchetkov R, Connor MK, Han K, et al. PKB/Akt phosphorylates p27, impairs nuclear import of p27 and opposes p27-mediated G1 arrest. Nat Med 2002;8:1153-1160.

93. Besson A, Gurian-West M, Schmidt A, Hall A, Roberts JM. p27Kip1 modulates cell migration through the regulation of RhoA activation. Genes Dev 2004;18:862-876. 